\title{
Non-canonical regulation of phosphatidylinositol 3-kinase gamma isoform activity in retinal rod photoreceptor cells
}

\author{
Vivek K Gupta ${ }^{1,4}$, Ammaji Rajala, ${ }^{1,4}$ and Raju VS Rajala1,2,3,4*
}

\begin{abstract}
Background: Phosphatidylinositol 3-Kinases (PI3Ks) are a family of lipid kinases that phosphorylate the D3-hydroxyls of the inositol ring of phosphoinositides, and are responsible for coordinating a diverse range of cellular functions. A canonical pathway of activation of PI3Ks through the interaction of RA-domain with Ras proteins has been well established. In retinal photoreceptors, we have identified a non-canonical pathway of PI3KY activation through the interaction of its RA-domain with a putative Ras-like domain (RLD) in alpha subunit of cyclic nucleotide-gated channel (CNGA1) in retinal rod photoreceptors.

Results: The interaction between PI3KY and CNGA1 does not appear to play a role in regulation of CNG channel activity, but PI3KY uses CNGA1 as an anchoring module to achieve close proximity to its substrate to generate D3-phosphoinositides.

Conclusions: Our studies suggest a functional non-canonical PI3KY activation in retinal rod photoreceptor cells.

Keywords: Cyclic nucleotide-gated channel, Phosphatidylinositol 3-kinase gamma, Photoreceptor outer segments, Ras-associating domain, Ras-like domain
\end{abstract}

\section{Introduction}

Phosphatidylinositol 3-Kinases (PI3Ks) are a family of lipid kinases that catalyze the phosphorylation of D3hydroxyls in the inositol head group and generate several phosphorylated phosphoinositides [1]. The D3phosphorylated products further act as membrane tethers to several phospholipid binding proteins which regulate a diverse range of cell functions, including proliferation, cell survival, degranulation, vesicular trafficking, and cell migration [1]. There is a highly conserved Ras-associating (RA) domain present in different isoforms of class I and class II PI3Ks [1]. A canonical pathway of the activation of PI3K through interaction of its RA-domain with Ras protein has been well established [2]. In retinal photoreceptors, we found a non-canonical pathway of PI3KY activation through interaction of its

\footnotetext{
* Correspondence: raju-rajala@ouhsc.edu

'Department of Ophthalmology, University of Oklahoma Health Sciences Center, 608 Stanton L. Young Boulevard, Oklahoma City, OK 73104, USA ${ }^{2}$ Department of Physiology, University of Oklahoma Health Sciences Center, 608 Stanton L. Young Boulevard, Oklahoma City, OK 73104, USA

Full list of author information is available at the end of the article
}

RA-domain with a putative Ras-like domain (RLD) in the cyclic nucleotide-gated channel alpha subunit (CNGA1) in retinal rod photoreceptors.

Photoreceptor cyclic nucleotide-gated (CNG) channels are critical elements in phototransduction and light adaptation $[3,4]$. They are responsible for generating the light response in photoreceptors and are directly and co-operatively gated by the availability of cGMP [3] Interestingly, PI3K-generated phosphoinositides have also been shown to modulate the olfactory $[5,6]$ and cone photoreceptor CNG [7] channels. However, the interaction between PI3KY and CNGA1 does not appear to play a role in regulation of CNG channel activity.

This first report of PI3Ky interaction with a Ras-like domain in the nucleotide binding region of CNGA1 provides a framework within which detailed kinetic and structural studies can be carried out to establish the detailed mechanism and functional relevance of this interaction. The functional regulation of $\mathrm{PI} 3 \mathrm{~K} \gamma$ by Gprotein coupled receptors [8], Ras [2], and CNG channels through its RA domain establishes the important position of PI3Ks in signal transduction and provides further 
evidence of how closely the cell signaling network is integrated with phototransduction.

\section{Results}

PI3K interacts with CNGA1

We previously reported that the C-terminal region of CNGA1 displays $50-70 \%$ tertiary structural similarity towards Ras proteins [9]. We named this region the Ras-like (RLD) domain. Our studies also suggest that growth factor receptor-bound protein 14 (Grb14), a Ras-associating (RA) domain-containing protein, binds to CNGA1 and modulates channel activity [10]. Our membrane yeast two-hybrid screens also identified PI3K as a CNGA1 interaction partner (data not shown).

The RA-domain is present in class IA (p110 $, \beta, \delta$ ), class IB (p110 $\gamma)$, and class II (PI3K-C2 $\alpha, \beta, \gamma)$ PI3Kisoforms [11]. However, class I (PI, PI-4-P, PI-4,5- $\mathrm{P}_{2}$ ) and class II isoform (PI, PI-4-P) substrate specificities are different in terms of PI-4-5- $\mathrm{P}_{2}$, as class II does not use this PI, but class I does. To determine the category of PI3K isoform associating with CNGA1, solubilized rod outer segments (ROS) were immunoprecipitated (IP) with anti-CNGA1 antibody or non-immune IgG (control), and changes in PI3K activity were evaluated using PI-4,5- $\mathrm{P}_{2}$ as substrate. The results indicate that PI3K activity was associated with anti-CNGA1 IPs; no activity was associated with control IPs (Figure 1A-B). This experiment also suggested that as the substrate PI4,5- $\mathrm{P}_{2}$ can only be utilized by class I PI3K, the PI3K associated with CNGA1 could be class I PI3K (either IA or IB), but could not be class II PI3K.
Expression of PI3K isoforms in retinal rod photoreceptors CNGA1 is expressed mainly in the plasma membranes of the rod photoreceptor outer segments [3]. To determine whether class I PI3K isoforms are expressed in rod outer segments (ROS), we immunoblotted ROS from dark- and light-adapted rats with anti-PI3K $\alpha$, anti-PI3K $\beta$, antiPI3K $\gamma$, anti-CNGA1, and anti-opsin antibodies. We found expression of all class I PI3K isoforms in ROS, irrespective of dark- or light-adaption (Figure 1D-F). The ROS specific proteins, CNGA1 and opsin, were used as internal controls (Figure 1C, G). The expression profile of PI3K $\delta$ in ROS was not evaluated, as its expression is restricted exclusively to leukocytes [12].

PI3Ky is associated with the CNGA1 in light-adapted ROS

To establish the differential involvement of class IA or class IB PI3K associated with the CNGA1 in light-adapted ROS (LROS), we specifically immunoprecipitated (IP) CNGA1 with anti-CNGA1 antibody. The IPs were washed and incubated with either buffer or DMSO (vehicle to dissolve PI3K inhibitors), or PI3KY inhibitor 1 and 2 (1: AS605240, 8nM, 2: AS604850, $250 \mathrm{nM}$ ), or PI3K $\alpha$ inhibitor 1 and 2 (1: PI-103, $8 \mathrm{nM}, 2$ : PI3K $\alpha$ inhibitor 2, $2 \mathrm{nM}$ ). We then carried out PI3K activity assays using PI-4,5- $\mathrm{P}_{2}$ as a substrate. Our results indicate that PI3K activity was inhibited by $85 \pm 5 \%$ upon treatment with inhibitors of $\gamma$ isoform, while for the $\alpha$-isoform activity was inhibited by $20 \pm 5 \%$, compared to either buffer or DMSO control (Figure 2). We found similar results with dark-adapted ROS (data not shown). These experiments largely highlight the association of the class IB PI3K $\gamma$ isoform with CNGA1.

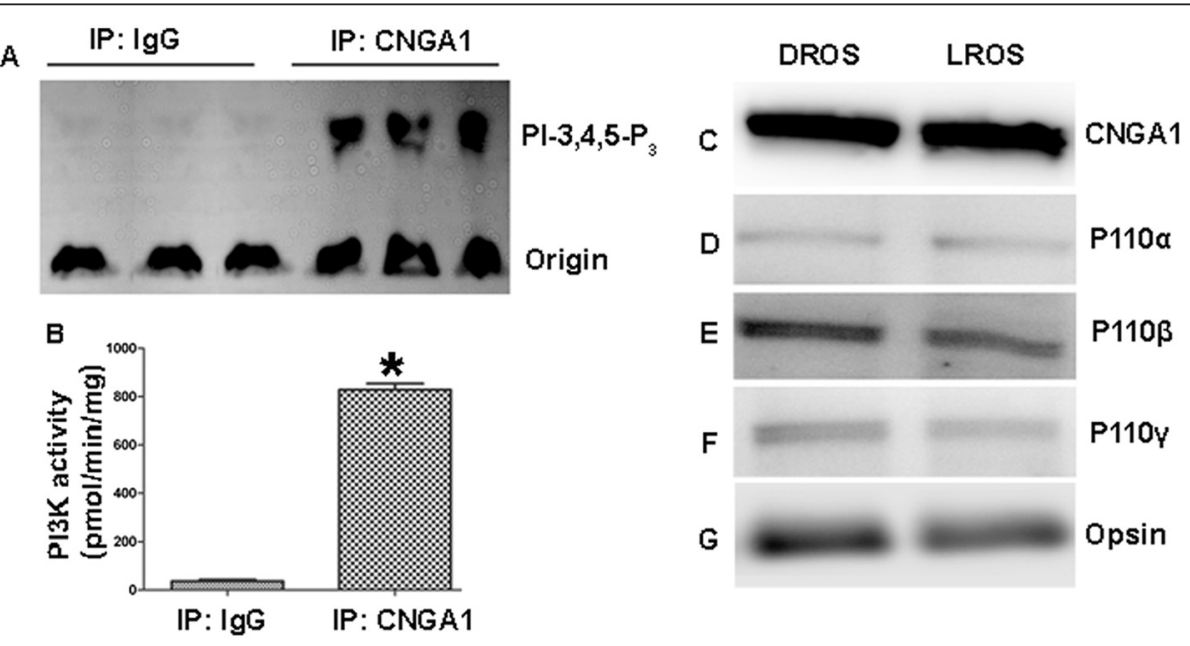

Figure 1 PI3K interacts with CNGA1 in vivo. PI3K enzyme activity was measured in anti-CNGA1 or non-immune lgG immunoprecipitates from solubilized ROS. PI3K activity was measured using PI-4,5- $\mathrm{P}_{2}$ and $\left[\gamma^{32} \mathrm{P}\right]$ ATP as substrates (A). The radioactive spots of PI-3,4,5- $\mathrm{P}_{3}$ were scraped from the TLC plate and counted (B). Data are mean $\pm S D(n=3),{ }^{*} p<0.001$. Immunoblot analysis of ROS proteins from dark-and light-adapted rats with anti-CNGA1(C), p110a (D), p110ß (E), p110y (F), and anti-opsin (G) antibodies. 


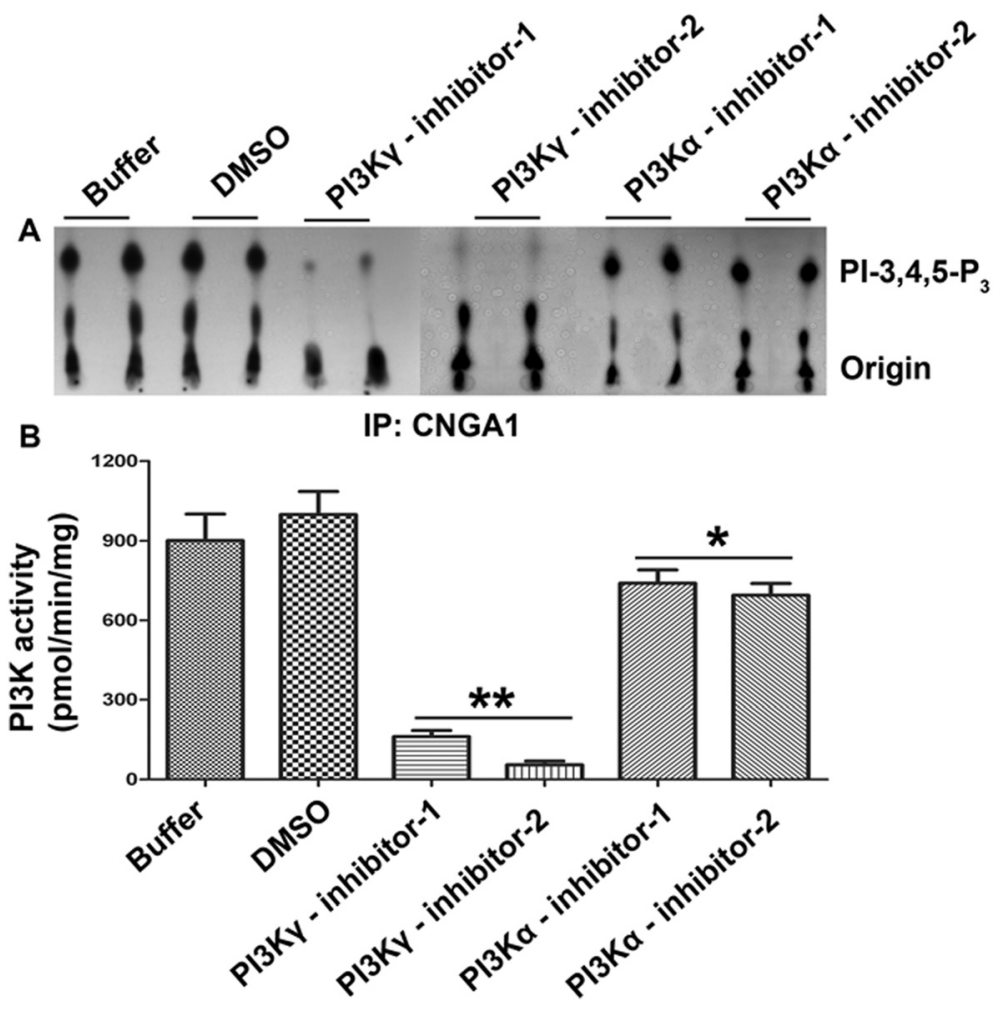

Figure 2 PI3KY isoform predominantly associates with CNGA1. Solubilized LROS was immunoprecipitated using anti-CNGA1 antibody and the washed immunoprecipitates were incubated with either buffer or DMSO or PI3KY inhibitor 1 and 2 or PI3Ka inhibitor 1 and 2 for 30 min. Then, the PI3K activity assay was carried out using PI-4,5- $\mathrm{P}_{2}$ and $\left[\gamma^{32} \mathrm{P}\right]$ ATP as substrates (A). The radioactive spots of PI-3,4,5-P 3 were scraped from the TLC plate and counted using scintillation counter (B). Data are mean $\pm S D(n=4) .{ }^{*} p<0.05,{ }^{* *} p<0.001$ (compared to buffer and DMSO control).

\section{CNGA1-associated $\mathrm{PI} 3 \mathrm{~K} \gamma$ activity is independent of dark and light conditions, as well as insulin receptor impairment in rods}

We previously reported a light-dependent association of PI3K $\alpha$ with tyrosine-phosphorylated IR in rod photoreceptor outer segments [13]. To determine whether light has any effect on the association of PI3K $\gamma$ with CNGA1 and to further rule out the involvement of IR signaling in regulating $\mathrm{PI} 3 \mathrm{~K} \gamma$ activation, we immunoprecipitated CNGA1 using anti-CNGA1antibody or normal IgG from the solubilized ROS of dark- and light-adapted mice, and from IR wild-type and IR knockout mice. PI3K activity was measured using PI-4,5- $\mathrm{P}_{2}$ as substrate. The results indicated that there was no significant difference between dark- and light-adapted (Figure 3A, B) or between IR wild-type and IR knockout (Figure 3C, D) mouse retinas, in terms of the association of PI3K $\gamma$ with CNGA1. These experiments clarify that, unlike activation of PI3K $\alpha$ which is light- and receptor tyrosine kinase IR-dependent [14], the association of $\mathrm{PI} 3 \mathrm{~K} \gamma$ with CNGA1 may be mediated through a simple proteinprotein interaction between the RA and RLD domains.

\section{PI3Kץ interacts with CNGA1 through its RA domain}

The regions encompassing the RA domain of PI3K $\alpha$ and $\mathrm{PI} 3 \mathrm{~K} \gamma$ were cloned as in frame fusion with a Myc-tag into pcDNA3 vector and co-expressed in HEK-293 T cells along with a Flag-tagged cytoplasmic domain of CNGA1 (CTR) in pcDNA3 vector. The RA domain of PI3K $\gamma$ was expressed with an additional sequence at the N-terminal (sequence showing homology with RA-PI3K $\alpha$ ) as the RA domain (220-311, [2]) clone was not observed to be stably expressed in HEK-293 T cells under the present conditions. The reciprocal immunoprecipitations with Myc and Flag antibodies revealed that interaction between CNGA1 and $\mathrm{PI} 3 \mathrm{~K} \alpha$ or $\mathrm{PI} 3 \mathrm{~K} \gamma$ is mediated through the interactions between complementary domains, the RA domain of PI3K and the RLD domain of CNGA1. The results also indicated that the RA domain of PI3K $\gamma$ interacts strongly with CTR-CNGA1, compared with the RA domain of PI3K $\alpha$ (100\% versus 30\%, Figure 4).

The interaction between the RA domains of PI3K $\alpha$ or PI3K $\gamma$ and CNGA1 were further evaluated by split ubiquitin-based membrane yeast two-hybrid analysis [10]. CNGA1 was observed to interact with RA-PI3K $\gamma$ 


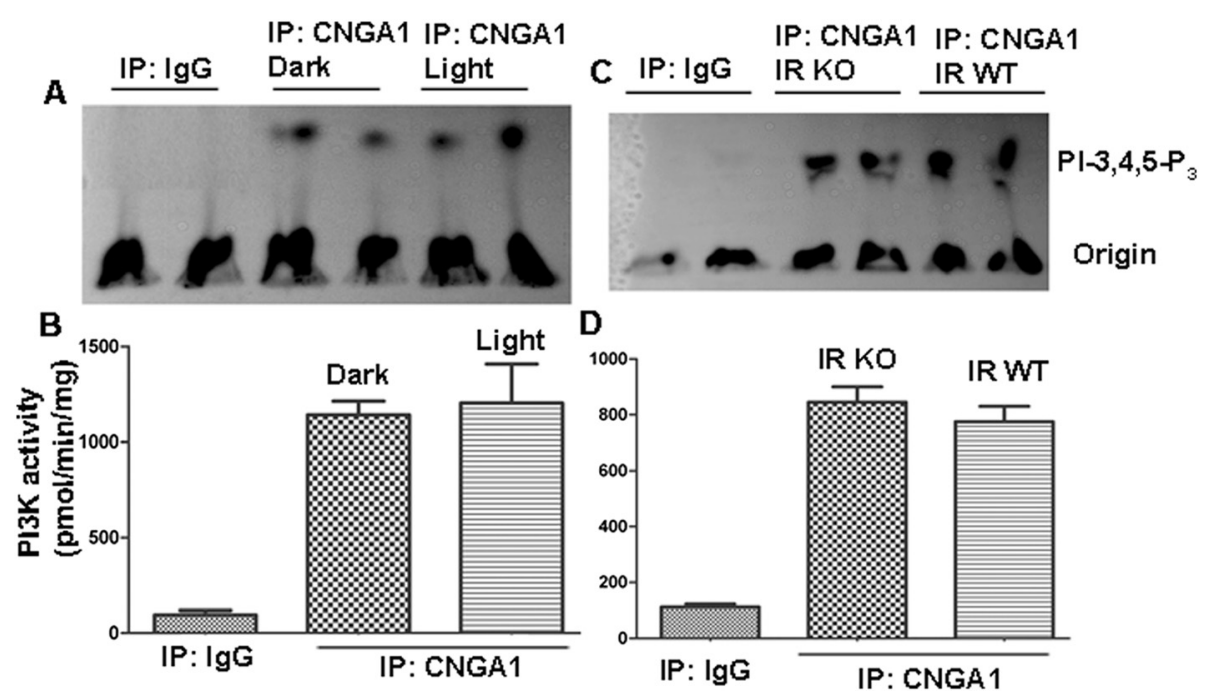

Figure 3 PI3K activity associated with CNGA1 is independent of light and IR activation. Solubilized ROS from either dark or light-adapted mice (A) or IR wild-type (C) or IR knockout (C) mice were immunoprecipitated with anti-CNGA1 antibody or non-immune IgG. PI3K activity was measured using Pl-4,5- $\mathrm{P}_{2}$ and $\left[\mathrm{\gamma}^{32} \mathrm{P}\right] \mathrm{ATP}$ as substrates. The radioactive spots of PI-3,4,5- $\mathrm{P}_{3}$ were scraped from the TLC plate and counted (B, D). Data are mean $\pm S D(n=3)$.

more strongly than the RA-PI3Ka isoform. The strength of the interaction was quantified by liquid $\beta$-galactosidase activity assays. We observed that RA-PI3K $\alpha$ derives the reaction to $25 \pm 10 \%$, compared to that of the RA-PI3KY isoform (Figure $5 \mathrm{C}$ and $\mathrm{D}$ ). The protein expression of CNGA1 in the yeast was confirmed by immunoblotting (Figure $5 \mathrm{~B}$ ). The CNGA1 was observed to target properly to the membrane, as it was localized exclusively to the membrane fraction of the yeast and absent in the cytosolic fraction (Figure 5A). G6PDH was used as a marker for the cytoplasm (Figure 5A). The results further support that the RA domain of PI3K $\gamma$ interacts strongly with CTRCNGA1, compared with the RA domain of PI3Ka.

\section{Effect of RA-PI3KY on channel activity}

The Myc-tagged RA domain of PI3K $\gamma$ was co-expressed along with the CNGA1 in HEK-293 T cells, and its effect on CNGA1 channel activity was measured [10]. It was observed that co-expression with RA-PI3KY does not lead to an alteration in the channel activity. CNGA1 cotransfected with pCDNA3 and RA-PI3K $\gamma$ alone were used as controls (Figure 6A). To confirm that the lack of any effect on channel activity is not due to the absence of PI3K $\gamma$ expression, we immunoblotted the expressed proteins with anti-CNGA1 and anti-Myc antibodies. The results established comparable levels of CNGA1 and RA-PI3K $\gamma$ expression (Figure 6B).

We observed that the RA domain of CNGA1 exhibits considerable tertiary structural homology with the RA domain of Grb14, which is known to inhibit CNGA1 activity, as shown in Figure 6C. RA-Grb14 inhibits CNGA1 through the involvement of Glu, ${ }^{180,} 181$ which is present at the $\beta$-turn with a unique orientation [15]. Our in silico studies identified that, in RA-PI3K $\gamma$, these Glu residues are present towards the $\beta$-sheet extension and their orientation does not allow them to interact adequately with the CNGA1 in a manner that would facilitate modulation of its activity (Figure 6D). These in silico predictions were verified by site-directed insertional mutagenesis, where two Glu residues were introduced at 300,301 positions between an Asn and a Gly residue (Figure 6E). This modification imparts $\mathrm{PI} 3 \mathrm{~K} \gamma$ with the capability to inhibit CNGA1 significantly, as predicted from the model and as shown experimentally in the Figure 6F. To confirm the expression of proteins, immunoblotting was performed with anti-CNGA1 and anti-Myc antibodies. The results show comparable levels of protein expression under experimental conditions (Figure 6G). Together, these experiments suggest that, unlike Grb14, PI3K $\gamma$ may use CNGA1 as an adapter for activation, rather than playing a role in the modulation of CNG channel activity.

\section{Identification of the interaction constraints of CTR-CNGA1 and RA-PI3KY}

The RA domain of Grb14 interacts with CNGA1 and Ras proteins through the involvement of Lys140 residue $[9,15]$ as shown (Figure 7A). The RA domain of PI3K $\gamma$ has also been shown to interact with Ras proteins through Lys residues $(251,255,256)$. We observed that the domain is located on a similar tertiary structural fold as shown in Figure 7B. These observations suggest that RA-PI3K $\gamma$ and CTR-CNGA1 interactions are potentially 

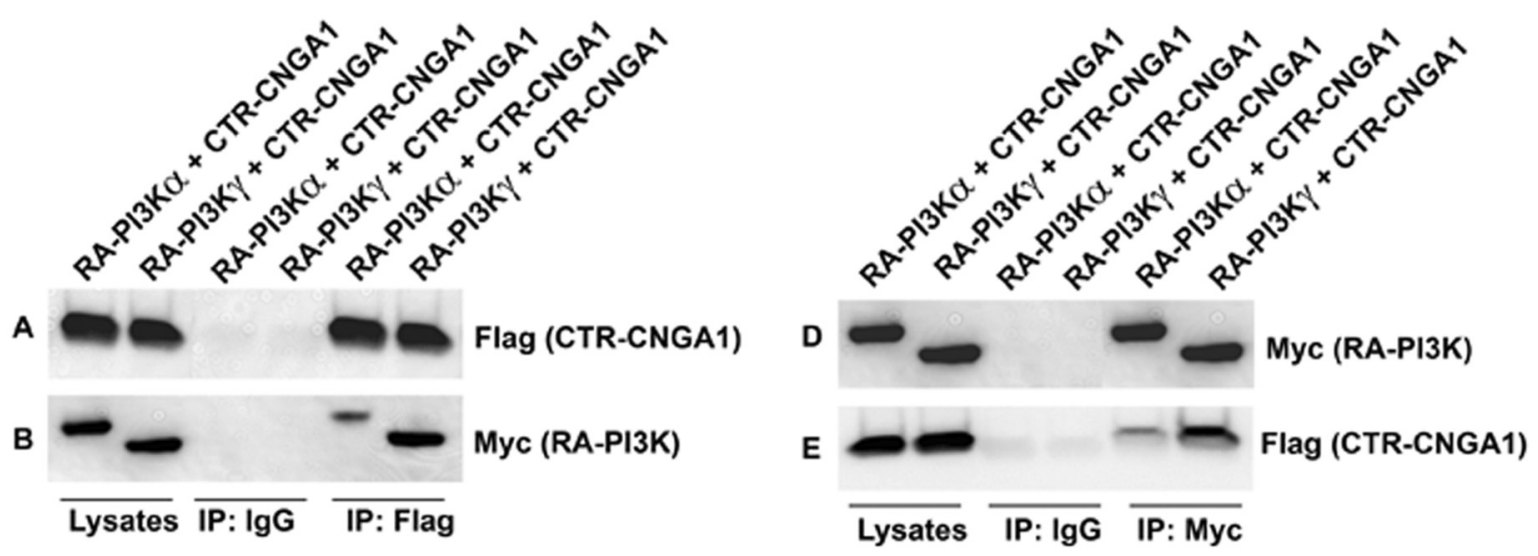

C
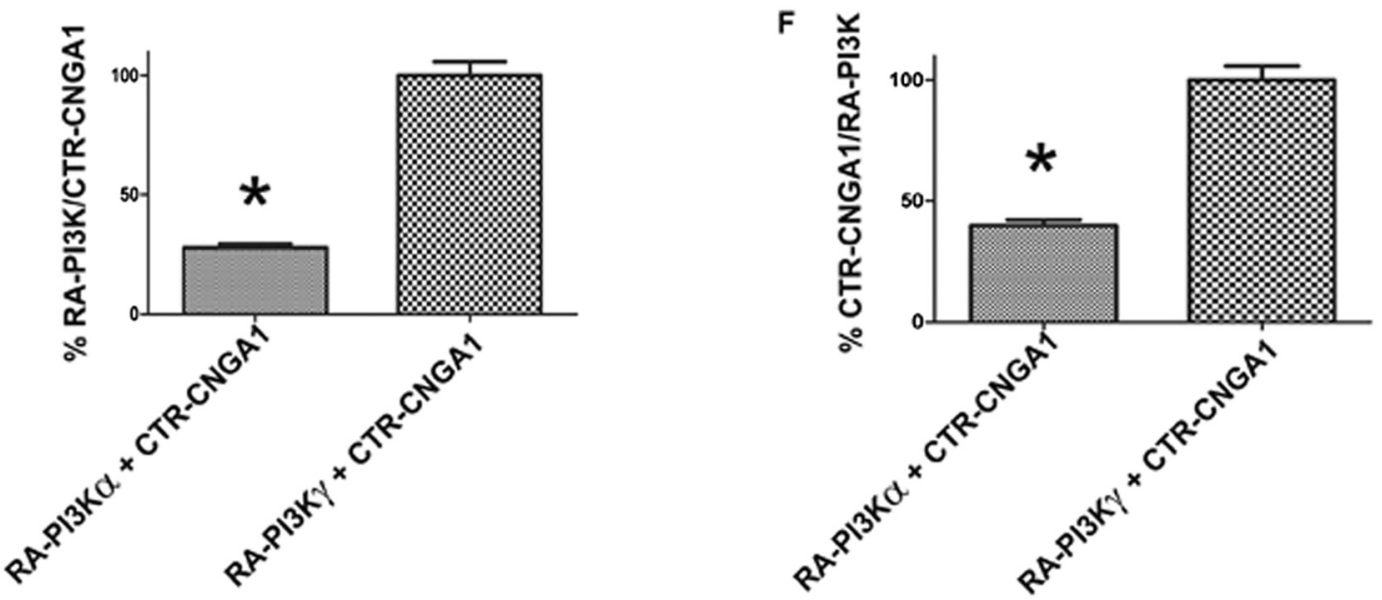

Figure 4 PI3Ka and $\gamma$ isoforms associate with CNGA1 through their RA domain. The physical interaction of these domains was analyzed by reciprocal co-immunoprecipitations. The Flag-tagged RLD-CNGA1 domain and Myc-tagged PI3Ka and $\gamma$ RA domains were transiently expressed in HEK-293 T cells. Expressed proteins were subjected to immunoprecipitation with either anti-Flag or anti-Myc antibodies or IgG. Anti-Flag and normal IgG IPs were subjected to immunoblot analysis with anti-Flag (A) and anti-Myc (B) antibodies along with Flag-and Myc-expressed protein lysates, whereas anti-Myc and normal IgG IPs were subjected to immunoblot analysis with anti-Myc (D) and anti-Flag (E) antibodies along with Myc- and Flag-expressed protein lysates. The relative binding was expressed as percentage of the RA-PI3K and RLD-CNGA1 and vice versa in each case $(\mathbf{C}, \mathbf{F})$. Data are mean $\pm S D, n=3,{ }^{*} p<0.001$.

mediated through Lys residues, which have protruded side chains and present a highly positively charged local environment to the protein motif. This region is more positively charged than the RA domain of Grb14, and its positive cloud is further enhanced by the $K^{254}$ residue which is present in its vicinity (Figure 7C). We previously reported that RA-domain and RLD interactions are electrostatic [9] and require complementary charges on the interacting partner. The surface topology of the modeled CTR-CNGA1 $[9,15]$ was probed for the distribution of negatively charged residues, which are easily accessible to the Lys residues of RA-PI3K $\gamma$. The most negatively charged pocket on the surface of the CTR-CNGA1 suggested a potential sink for the most favorable interaction that may exist between these two domains. Molecular modeling revealed that K251, 255, and 256 forms a potential electrostatic interaction with the D577, D578, and E581 located on the surface of cytoplasmic region of CNGA1. Although E488 is not directly involved in interactions, it may still contribute to the formation of a negatively charged cloud. The complete view is shown in Figure 7C. The predictions made by molecular modeling were confirmed by site-directed mutagenesis, followed by the overexpression of the proteins in the HEK293 T cells (Figure 7D-G). Reciprocal co-immunoprecipitations of the tagged proteins revealed that the physical interaction was completely broken upon the mutation of all three residues: D577, D578, and E581 to N, N, and Q, respectively (Figure $7 \mathrm{H}$ ). The individual or double mutation of the residues could not completely alleviate the interaction. Densitometric quantification revealed that the E581Q mutation can break the interaction by $50 \pm 10$ 


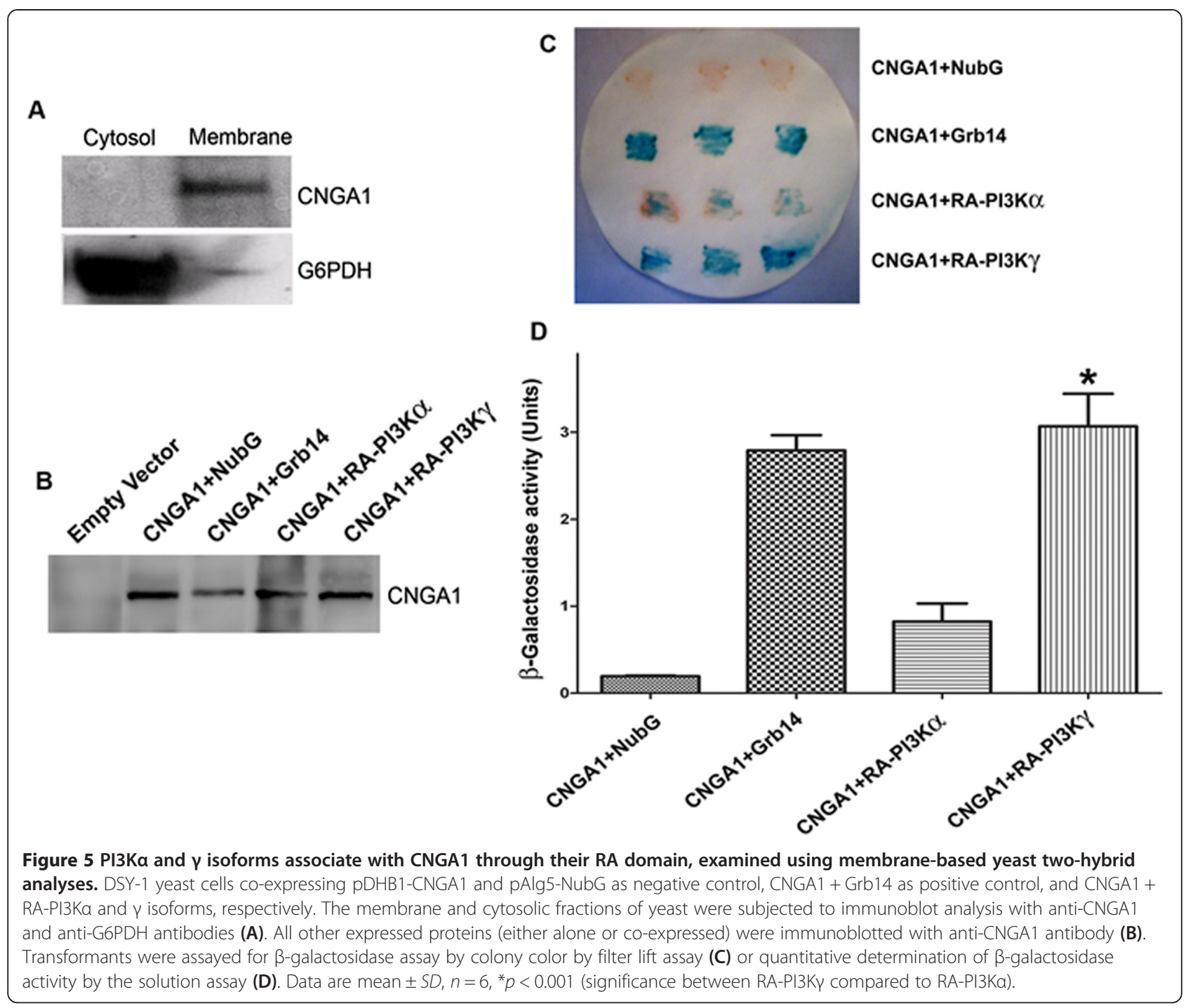

percent. D577N disrupted the interaction by $30 \pm 10$ percent, while an interaction disruption of $45 \pm 10$ percent was observed with a double mutation of D577, $578 \mathrm{~N}$.

\section{Discussion}

Ras proteins form a direct contact with the PI3K $\gamma$ catalytic domain. These unique interactions are most likely also shared by the Ras/PI3K $\alpha$ isoform [2]. Consistent with earlier findings, in the present study we found that the RA-domain of PI3K $\gamma$ associated with a Ras-like domain (RLD) in CNGA1 channel subunit. Our results further suggest that the RA-domain of PI3K $\gamma$ binds to the RLD region of CNGA1 significantly more strongly than does the RA-domain of PI3K $\alpha$. We also found that the $\mathrm{RA}$-domain of PI3K $\gamma$ has no effect on channel activity, suggesting that PI3K $\gamma$ may tether to CNGA1 for its activation (Figure 8). It is interesting to note that the RAdomain of Grb14 can modulate the channel activity, but not the RA-domain of PI3K $\gamma$. We previously reported that Grb14 competes with cGMP for the CNGA1 binding pocket, and electrostatically interacts with $\mathrm{Arg}^{559}$ through a negatively charged $\beta$-turn at its RA domain [15]. We showed that three Glu residues (180-182) in Grb14 are critical for electrostatic interaction with the cGMP binding pocket and the resultant inhibition [15]. Tertiary structural analysis revealed that the RA domain of Grb14 shows a $50-60 \%$ structural similarity with the RA domain of PI3K $\gamma$, with the position of residues and arrangement of most secondary structural motifs conserved. The RA domain of PI3K $\alpha$ is not significantly similar in structure to the RA domains of either PI3K $\gamma$ or Grb14.

Examination of the RA domains in PI3K $\gamma$, PI3K $\alpha$, and Grb14 suggests a molecular divergence among the RA domains in intracellular signaling proteins. We attributed this difference between RA-PI3K $\gamma$ and RA-Grb14's 


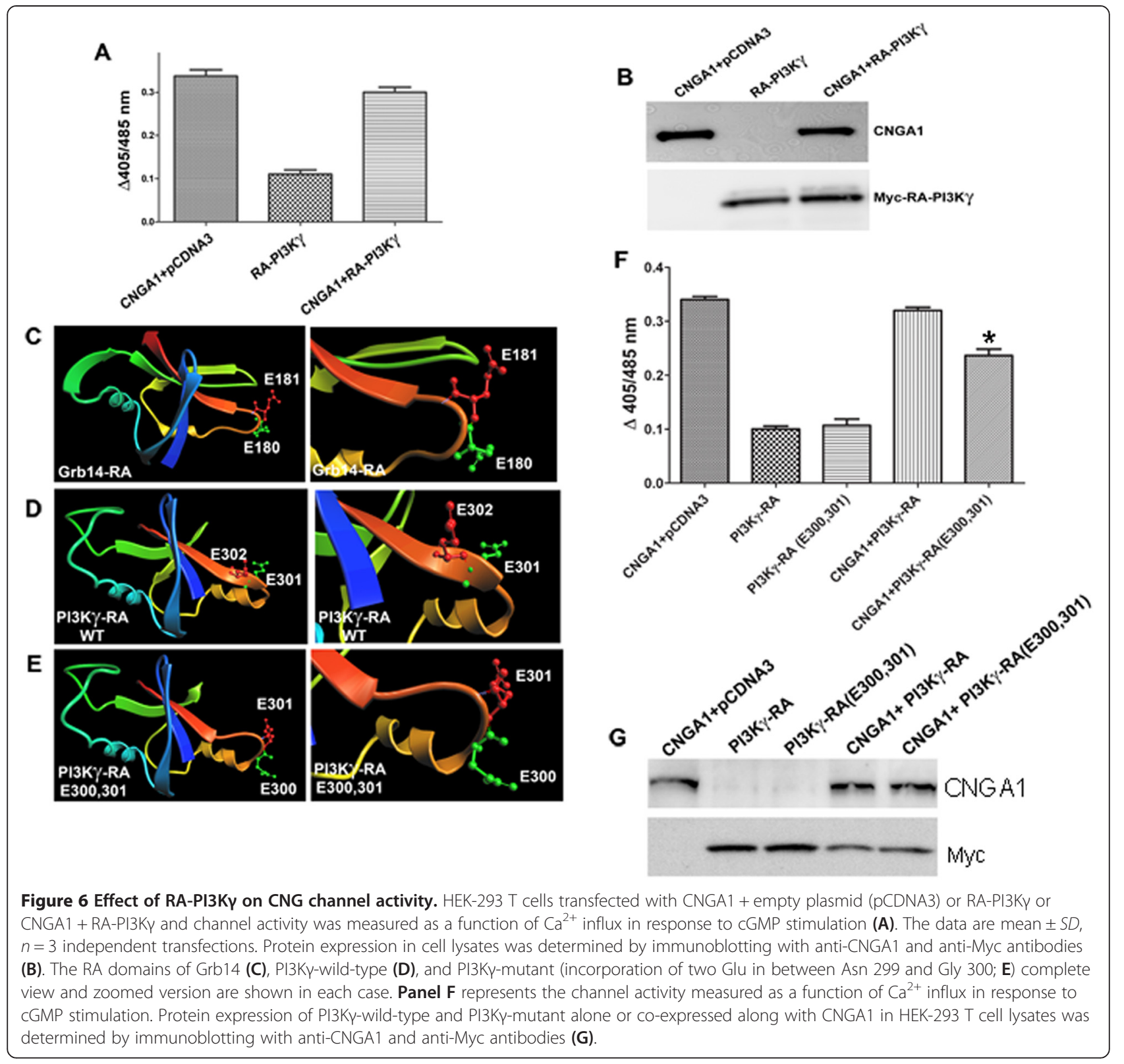

inhibitory effects on the channel to the orientation of the Glu residues in the $\beta$-turn. We confirmed this hypothesis by site-directed insertional mutagenesis in which two Glu residues were introduced at the 300,301 positions between an Asn and a Gly residue, and found a significant inhibition of channel activity by RA-PI3K $\gamma$.

The fraction of the total PI3K $\gamma$ bound to CNGA1 in photoreceptors is unknown. Nevertheless, it must constitute an important proportion of the total pool, as the alternative Ras-mediated pathway is insufficient to drive membrane translocation of PI3K $\gamma$, a necessity for its activation [16]. Alternatively, this binding might complement the Ras-PI3K $\gamma$ interaction pathway by accepting the allosterically activated PI3K from Ras and enabling it to stay bound at the membrane. The interaction of CNGA1 with PI3KY also indicates that CNGA1 may contribute towards the existence of an alternative pathway to Ras activation of PI3K $\gamma$, strengthened by the fact that both CNGA1 and Ras proteins are nucleotide binding proteins showing similar structural folds. The relative contribution of these controlling mechanisms, as well as the fraction of the $\mathrm{PI} 3 \mathrm{~K} \gamma$ participating in each of the regulatory interactions, is yet to be determined in vivo.

The crystal structure of PI3K $\gamma$ in complex with Ras has revealed that $\mathrm{K}^{251,254,255}$, and ${ }^{256}$ are critical residues for establishing an interaction with Ras [2]. Lys ${ }^{140}$ of Grb10 [17] and Grb14 [15] have been shown to be 

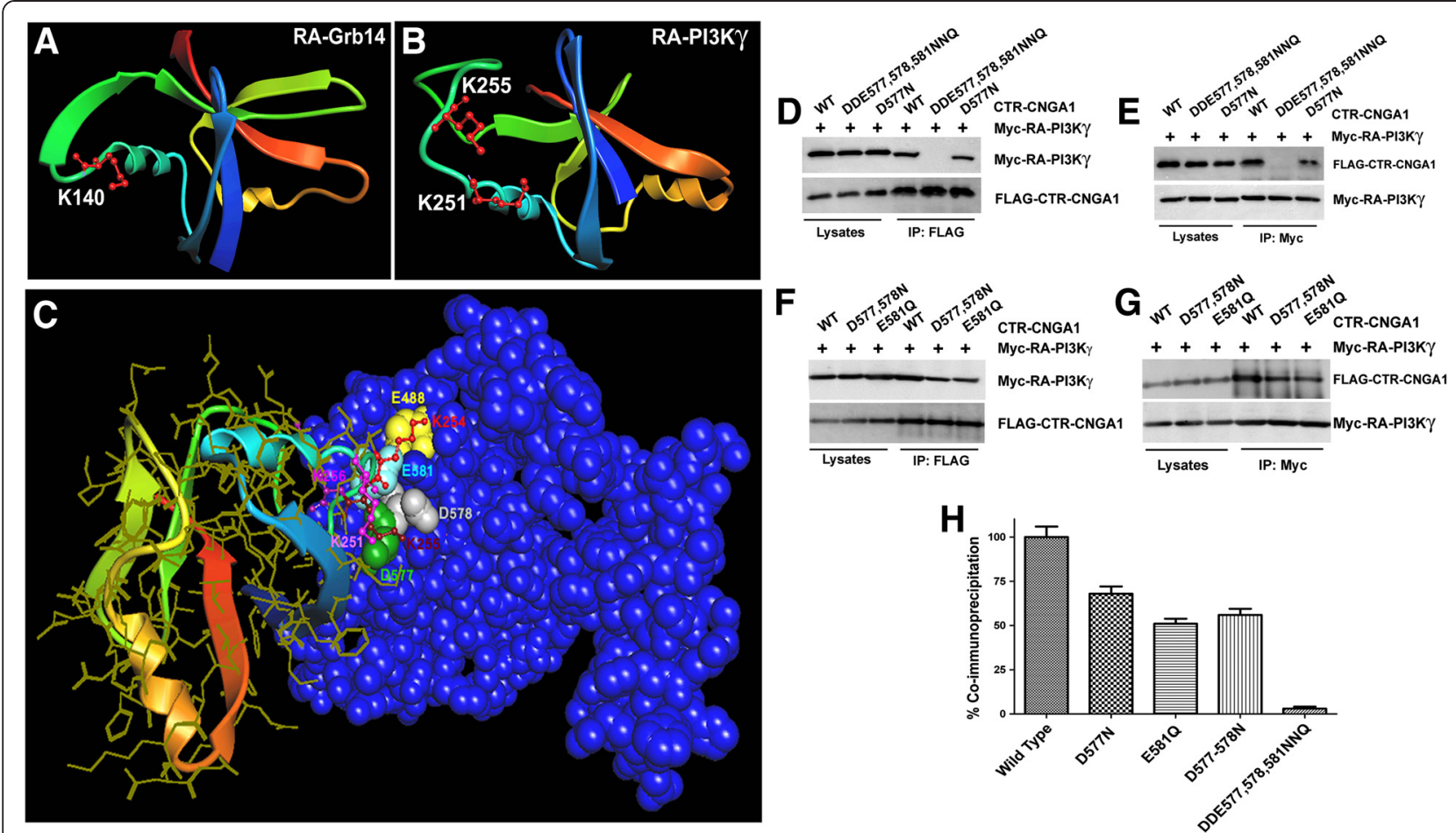

Figure 7 Molecular determinants involved in RA-PI3Ky interaction with CNGA1. Ribbon-shaped model of the RA domain of Grb14 [UniProtKB Accession No. Q5ICW4] (106-192) constructed using the RA domain of Grb10 as a template (PDB ID: 3HK0). The interacting residues of RA-Grb14 (A) and RA-PI3KY (B) are highlighted as ball and stick (red) and labeled. The corresponding secondary structural motifs of the two structures are similarly colored. Complete view of the interaction between RA-PI3Ky (ribbon, multicolored) and the cytoplasmic domain of CNGA1 (CPK, blue; C). The positively charged random coil element of RA-PI3KY with projecting Lys side chains (251 pink, 255 brown, 256 purple and 254 red; ball and stick, labelled) interacts with $\mathrm{Asp}^{577}$ (green space-filled and labeled), Asp578 (gray space-filled and labeled) and Glu581 (light blue, space-filled and labelled). Glu488 (yellow, space-filled and labeled) provides additional anchorage through the interaction with K254. The interacting residues of RA-PI3KY are highlighted as ball and stick and labeled, while the CNGA1 interacting residues are represented as space-filled structures and labeled. We investigated the interaction between RLD-CNGA1 and its mutant proteins by subjecting the HEK-293 T cell lysates expressing respective proteins to immunoprecipitation with anti-Flag antibody (D), followed by immunoblotting and probing for RA-PI3KY with anti-Myc antibody (D, F) and vice versa $(\mathbf{E}, \mathbf{G})$. Cell lysates were also loaded in each case for comparison. The interaction was quantified by densitometric analysis of the band intensities in immunoblots, performed in the linear range of detection, and normalized by the total amount of RLD-CNGA1 or RA-PI3KY immunoprecipitated in each case $\mathbf{( H )}$. The relative values were expressed as percentage of the co-immunoprecipitation in wild-type proteins. Data are mean $\pm S D, n=3$, significance between each of the wild type RLD-CNGA1, D577N, E581Q, D577-578 N, and DDE 577,578, 581 NNQ * $p<0.001$.

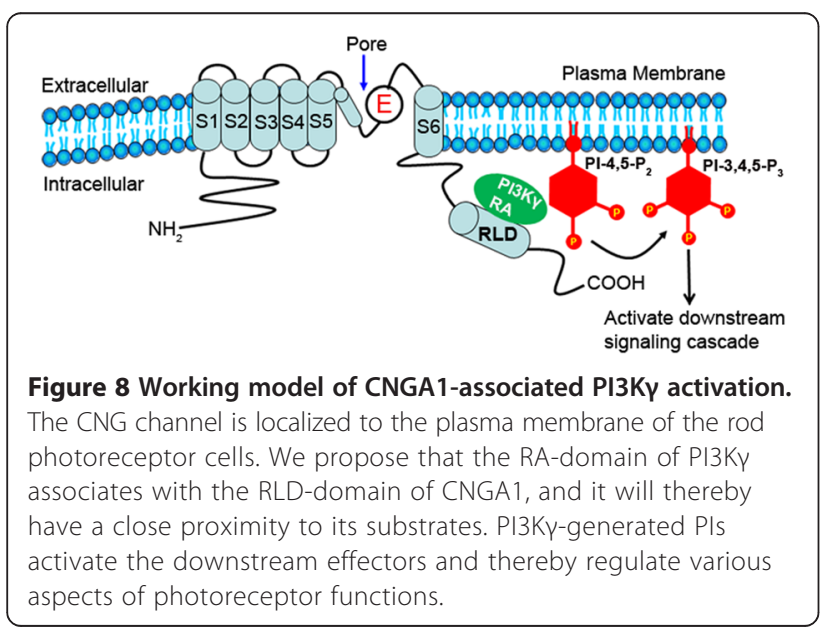

involved in the interaction of the RA domain with Ras proteins and the Ras-like domain of CNGA1. Lys ${ }^{140}$ of Grb14 corresponds to the Lys ${ }^{251}$ and Lys $^{255}$ of the RAPI3K $\gamma$, but is present amongst a highly positively charged cloud along the $\beta$-turn, imparting it with more leverage to interact and associate with proteins carrying negatively charged surface pockets. These types of electrostatic interactions comprise the basis of PI3Ky's interaction with Ras proteins [2]. These interactions may also be a good model for the weak or potentially transient interaction of RA-PI3K $\alpha$ with CNGA1. In addition, these interactions might contribute to conformational changes in PI3K $\gamma$, similar to those caused by Ras protein binding [2] and leading to altered PI3K $\gamma$ activity and membrane recruitment. The structural folds of the Ras-associating domain of PI3K $\gamma$ 
are also similar to Raf and RalGDS, two other effectors of Ras proteins [18,19].

The phospholipids have been shown to play significant roles in the negative modulation of CNG channels [20-23]. Even though PI3K $\gamma$ may not have a direct effect on CNG channel modulation, PI3K $\gamma$-generated PI-3,4,5$\mathrm{P}_{3}$ may indirectly influence the channel activity. Accordingly, PI-3,4,5-P3 has been previously shown to inhibit the action of olfactory [5] and cone CNG [7] channels. Our studies thus suggest a functional non-canonical PI3K $\gamma$ activation in retinal rod photoreceptor cells.

\section{Materials and methods}

\section{Cell lines and culture conditions}

HEK-293 T cells were maintained at $37^{\circ} \mathrm{C}$ in DMEM medium containing $10 \%$ (v/v) FBS. Approximately $2.5 \times 10^{5}$ cells were seeded in each 60-mm culture dish 12-18 h before transfection. Calcium phosphate-mediated DNA transfection was performed using each of the plasmids containing the cDNA of interest [24]. Cells were harvested for experiments $\sim 48 \mathrm{~h}$ post-transfection.

\section{Plasmids and DNA}

The regions encompassing the RA domain of PI3K $\alpha$ (Uniprot id. P42336, residues 132-314) and PI3KY isoform (P48736, residues 141-311; Open Biosystems, Huntsville, AL, USA) were amplified from their respective full-length PI3Ks and cloned into Myc-tagged pCDNA3 vector with Bam H1 and Xho1 sites. The CNGA1 channel antibodies were a kind gift from Dr. Robert Molday, University of British Columbia (Canada). The Myc-tagged CNGA1 construct was generated from the amplification of pCDNA3-CNGA1 with sense (GAA TTC ACC ATG GAG CAA AAA CTC ATC TCA GAA GAG GAG GAT CTG ATG AAG AAA GTG ATT ATC AAT ACA TGG CAC ) and antisense (CTC GAG TCA GTC CTG TGT AGA GTC TGT GGG CCC ACT TTC) primers. The $\mathrm{NH}_{2}$-terminal FLAG-tagged C-terminal region of CNGA1 (CTR-CNGA1,residues 483-690) was cloned into pCDNA3 using sense (GAA TTC ACC ATG GAT TAC AAG GAT GAC GAC GAT AAG GCT TGG TCT GTT GGT GGAG) and antisense (CTC GAG TCA GTC CTG TGT AGA GTC TGT GGG CCC ACT TTC) primers. The cDNA encoding the full-length CNGA1 was cloned into the NcoI site of pDHB1 membrane-based yeast two-hybrid bait vector. Full-length Grb14, RAPI3K $\gamma$, and RA-PI3K $\alpha$ isoforms were cloned as BamH1/ Sal1 into pDL2-Nx prey vector. The positive (pMBV-Alg5 and pAlg5-NubI) and negative (pMBV-Alg5 and pAlg5NubG) plasmids were obtained from Dualsystems Biotech AG (Switzerland). The membrane yeast two-hybrid assay was carried out using the Dual hybrid kit (Dualsystems Biotech AG, Switzerland). The membrane yeast twohybrid screen was used in the S. cerevisiae strains DSY-1.
Site-directed mutagenesis (SDM) was carried out according to the method described earlier [25]. The primers used for SDM were PI3K $\gamma$-RA (E 300, 301; sense: TGC CTC AAG AAC GAA GAA GGA GAA GAG ATT; antisense AAT CTC TTC TCC TTC TTC GTT CTT GAG GCA), CTR-CNGA1(DDE 577, 578, 581 NNQ; sense: TCA AAA AAT ACC CTC ATG CAA GCT CTA ACT; antisense: AGT TAG AGC TTG CAT GAG GTT ATT TTT TGA), CTR-CNGA1 (D577N; sense: CTC TCA AAA AAT GAC CTC ATG GAA GCT CTA; antisense: TAG AGC TTC CAT GAG GTC ATT TTT TGA GAG), CTR-CNGA1 (D577,578 N; sense: CTC TCA AAA AAT AAC CTC ATG GAA GCT CTA; antisense: TAG AGC TTC CAT GAG GTT ATT TTT TGA GAG), CTR-CNGA1 (E581Q; sense: AAA GAT GAC CTC ATG CAA GCT CTA ACT GAG; antisense: CTC AGT TAG AGC TTG CAT GAG GTC ATC TTT). After sequencing, the mutant cDNAs were excised from the sequencing vector and cloned into pCDNA3 mammalian expression vector.

\section{Animals}

All animal work was performed in strict accordance with the NIH Guide for the Care Use of Laboratory Animals, and the Association for Research in Vision and Ophthalmology on the Use of Animals in Vision Research. All protocols were approved by the IACUC of the University of Oklahoma Health Sciences Center and the Dean McGee Eye Institute. The generation of rod-specific conditional IR knockout mice has been reported previously [26]. A breeding colony of Albino Sprague-Dawley rats is maintained in our vivarium in cyclic light $(12 \mathrm{~h}$ on/ off; 300 lux). Experiments were carried out on male and female rats and mice.

\section{Membrane-based yeast two-hybrid assay}

Yeast transformations were performed using lithium acetate [25]. We used the split ubiquitin system to genetically investigate interactions between membrane proteins $[27,28]$ to probe the possible intercommunications between full-length CNGA1 and either of the full-length Grb14, RA-PI3K $\alpha$, or RA-PI3K $\gamma$, using the transcription factor protein-LexA-VP16 (PLV) as the reporter molecule. The filter paper $\beta$-galactosidase assay for the detection of interactions and liquid culture assay for their quantification was performed as described previously [29].

\section{PI3K activity assays}

Mouse and rat photoreceptor outer segments (ROS) were prepared as described previously [13]. Enzyme assays were performed on solubilized ROS, essentially as described previously [30]. Briefly, assays were performed directly on anti-CNGA1 immunoprecipitates in $50 \mu \mathrm{l}$ of the reaction mixture containing $0.2 \mathrm{mg} / \mathrm{ml} \mathrm{PI}-4,5-\mathrm{P}_{2}$, 
$50 \mu \mathrm{M}$ ATP, $0.2 \mu \mathrm{Ci}\left[\gamma^{32} \mathrm{P}\right]$ ATP (Perkin Elmer, MA, USA), $5 \mathrm{mM} \mathrm{MgCl}$, and $10 \mathrm{mM}$ HEPES buffer (pH 7.5). The reaction was performed for $30 \mathrm{~min}$ at $25^{\circ} \mathrm{C}$ and was stopped by the addition of $100 \mu \mathrm{l}$ of $1 \mathrm{~N} \mathrm{HCl}$ followed by $200 \mu \mathrm{l}$ chloroform-methanol $(1: 1 \mathrm{v} / \mathrm{v})$. Lipids were extracted and resolved on TLC plates (silica gel 60) with a solvent system of 2-propanol/2 $\mathrm{M}$ acetic acid $(65 / 35, \mathrm{v} / \mathrm{v})$. The plates were coated in $1 \%(\mathrm{w} / \mathrm{v})$ potassium oxalate in $50 \%(\mathrm{v} / \mathrm{v})$ methanol and then baked in the oven at $100^{\circ} \mathrm{C}$ for $1 \mathrm{~h}$ before use. TLC plates were exposed to $\mathrm{X}$-ray film overnight at $-70^{\circ} \mathrm{C}$. Radioactive lipids were scraped and quantified by liquid scintillation counting.

\section{Assessment of the channel activity by ratiometric measurement of $\left[\mathrm{Ca}^{2+}\right]_{i}$}

The fluorescent indicator Indo-1/AM was used to monitor $\mathrm{Ca}^{2+}$ influx through the CNGA1 channels in cell suspensions. The assays were performed as described $[10,15]$ using a spectrofluorometer (Fluostar Omega, BMG lab tech $\mathrm{GmBH}$, Offenburg, Germany). This assay was designed to determine CNG channel activity in cell populations $\left(2 \times 10^{6}\right)$ in response to 8 -pCPT-cGMP stimulation. Briefly, cells (36-48 h post-transfection) were harvested with cell dissociation medium (Invitrogen, Carlsbad, CA), washed with the extracellular solution (ECS; $140 \mathrm{mM}$ $\mathrm{NaCl}, 5 \mathrm{mM} \mathrm{KCl}, 1 \mathrm{mM} \mathrm{MgCl}, 1.8 \mathrm{mM} \mathrm{CaCl}, 10 \mathrm{mM}$ glucose, $15 \mathrm{mM}$ HEPES, $\mathrm{pH}$ 7.4), and incubated with $2 \mu \mathrm{M}$ Indo-1/AM (Sigma-Aldrich) in ECS in the presence of $0.05 \%$ Pluronic F-127 (Invitrogen, Carlsbad, CA) for $40 \mathrm{~min}$ at room temperature. Then, the cells were washed three times with ECS and resuspended in ECS $\left(1 \times 10^{6} / \mathrm{mL}\right) . \mathrm{Ca}^{2+}$ influx in response to 8 -pCPTcGMP was determined by ratiometric measurement, which represents the free intracellular $\mathrm{Ca}^{2+}$ concentration. Changes of intracellular $\mathrm{Ca}^{2+}$ concentration were expressed as a $\Delta 405 / 485$ ratio.

\section{Molecular modeling studies}

The protein tertiary structural modeling was done using the MODELLER program [31]. Structural manipulations, surface topology studies, and graphical representations were performed using Arguslab (Thompson, Planaria software), VMD [32], and DaliLite v3 [33]. The extensive energy minimization was done using Deep View [34]. Structural constraints and prediction quality of the modeled structures were evaluated using WHATIF [35] and PROCHECK [36].

\section{Statistical methods}

Data were analyzed and graphed using Graphpad Prism software (GraphPad Software, San Diego, CA). The data were expressed as the mean $\pm S D$ and compared by Student's $t$ test for unpaired data. The critical level of significance was set at $p<0.05$.

\section{Competing interests}

The authors declare that they have no competing interests.

\section{Authors' contributions}

Project planning, data analysis, and manuscript composition were performed by VK and RR. Experimental work was performed by VK, AR and RR. All authors read and approved the final manuscript.

\section{Acknowledgements}

This work was supported by grants from the NIH/NEI (EY016507; EY00871; EY021725) and an unrestricted grant from Research to Prevent Blindness, Inc. to the Department of Ophthalmology. The authors acknowledge Ms. Kathy J. Kyler, Staff Editor, Office of Research Administration, University of Oklahoma Health Sciences for editing this manuscript.

\section{Author details}

${ }^{1}$ Department of Ophthalmology, University of Oklahoma Health Sciences Center, 608 Stanton L. Young Boulevard, Oklahoma City, OK 73104, USA. ${ }^{2}$ Department of Physiology, University of Oklahoma Health Sciences Center, 608 Stanton L. Young Boulevard, Oklahoma City, OK 73104, USA.

${ }^{3}$ Department of Cell Biology, University of Oklahoma Health Sciences Center, 608 Stanton L. Young Boulevard, Oklahoma City, OK 73104, USA. ${ }^{4}$ Dean A. McGee Eye Institute, 608 Stanton L. Young Boulevard, Oklahoma City, OK 73104, USA.

Received: 23 November 2014 Accepted: 20 January 2015

Published online: 03 February 2015

\section{References}

1. Fruman DA, Meyers RE, Cantley LC. Phosphoinositide kinases. Annu Rev Biochem. 1998;67:481-507.

2. Pacold ME, Suire S, Perisic O, Lara-Gonzalez S, Davis $C T$, Walker EH, et al. Crystal structure and functional analysis of Ras binding to its effector phosphoinositide 3-kinase gamma. Cell. 2000;103:931-43.

3. Kaupp UB, Seifert R. Cyclic nucleotide-gated ion channels. Physiol Rev. 2002;82:769-824

4. Yau KW, Hardie RC. Phototransduction motifs and variations. Cell. 2009;139:246-64.

5. Brady JD, Rich ED, Martens JR, Karpen JW, Varnum MD, Brown RL. Interplay between Pip3 and calmodulin regulation of olfactory cyclic nucleotide-gated channels. Proc Natl Acad Sci U S A. 2006;103:15635-40.

6. Brunert D, Klasen K, Corey EA, Ache BW. Pi3kgamma-dependent signaling in mouse olfactory receptor neurons. Chem Senses. 2010;35:301-8.

7. Bright SR, Rich ED, Varnum MD. Regulation of human cone cyclic nucleotide-gated channels by endogenous phospholipids and exogenously applied phosphatidylinositol 3,4,5-trisphosphate. Mol Pharmacol. 2007;71:176-83.

8. Suire S, Condliffe AM, Ferguson GJ, Ellson CD, Guillou H, Davidson K, et al. Gbetagammas and the Ras binding domain of P110gamma are both important regulators of $\mathrm{Pi}(3)$ Kgamma signalling in neutrophils. Nat Cell Biol. 2006:8:1303-9.

9. Rajala RV, Rajala A, Gupta VK. Conservation and divergence of Grb7 family of Ras-binding domains. Protein Cell. 2012;3:60-70.

10. Gupta VK, Rajala A, Daly RJ, Rajala RV. Growth factor receptor-bound protein 14: a new modulator of photoreceptor-specific cyclic-nucleotide-gated channel. EMBO Rep. 2010;11:861-7.

11. Rajala RV. Phosphoinositide 3-kinase signaling in the vertebrate retina. J Lipid Res. 2010;51:4-22.

12. Fung-Leung WP. Phosphoinositide 3-kinase delta (Pi3kdelta) in leukocyte signaling and function. Cell Signal. 2011;23:603-8.

13. Rajala A, Anderson RE, JX M, Lem J, Al Ubaidi MR, Rajala RV. G-protein-coupled receptor rhodopsin regulates the phosphorylation of retinal insulin receptor. J Biol Chem. 2007;282:9865-73.

14. Rajala RV, Mcclellan ME, Ash JD, Anderson RE. In vivo regulation of phosphoinositide 3-kinase in retina through light-induced tyrosine phosphorylation of the insulin receptor beta-subunit. J Biol Chem. 2002;277:43319-26.

15. Gupta V, Rajala A, Rodgers K, Rajala RV. Mechanism involved in the modulation of photoreceptor-specific cyclic nucleotide-gated channel by The tyrosine kinase adapter protein Grb14. Protein Cell. 2011;2:906-17. 
16. Suire S, Hawkins P, Stephens L. Activation of phosphoinositide 3-kinase gamma by Ras. Curr Biol. 2002;12:1068-75.

17. Depetris RS, Wu J, Hubbard SR. Structural and functional studies of the Ras-associating and pleckstrin-homology domains of Grb10 and Grb14. Nat Struct Mol Biol. 2009;16:833-9.

18. Nassar N, Horn G, Herrmann C, Scherer A, Mccormick F, Wittinghofer A. The 2.2 A crystal structure of the Ras-binding domain of the serine/threonine kinase C-raf1 in complex with Rap1a and a Gtp analogue. Nature. 1995;375:554-60

19. Walker EH, Perisic O, Ried C, Stephens L, Williams RL. Structural insights into phosphoinositide 3-kinase catalysis and signalling. Nature. 1999;402:313-20.

20. Womack KB, Gordon SE, He F, Wensel TG, Lu CC, Hilgemann DW. Do phosphatidylinositides modulate vertebrate phototransduction? J Neurosci. 2000;20:2792-9.

21. Nilius B, Mahieu F, Prenen J, Janssens A, Owsianik G, Vennekens R, et al. The Ca2 +-activated cation channel Trpm4 is regulated by phosphatidylinositol 4,5-biphosphate. EMBO J. 2006;25:467-78.

22. Suh $B C$, Hille B. Regulation of ion channels by phosphatidylinositol 4,5-bisphosphate. Curr Opin Neurobiol. 2005;15:370-8.

23. He F, Mao M, Wensel TG. Enhancement of phototransduction G proteineffector interactions by phosphoinositides. J Biol Chem. 2004;279:8986-90.

24. Wigler M, Pellicer A, Silverstein S, Axel R. Biochemical transfer of single-copy eucaryotic genes using total cellular DNA as donor. Cell. 1978;14:725-31.

25. Rajala RV, Mcclellan ME, Chan MD, Tsiokas L, Anderson RE. Interaction of the retinal insulin receptor beta-subunit with the P85 subunit of phosphoinositide 3-kinase. Biochemistry. 2004;43:5637-50.

26. Rajala A, Tanito M, Le YZ, Kahn CR, Rajala RV. Loss of neuroprotective survival signal in mice lacking insulin receptor gene in rod photoreceptor cells. J Biol Chem. 2008;283:19781-92.

27. Johnsson N, Varshavsky A. Split ubiquitin as a sensor of protein interactions in vivo. Proc Natl Acad Sci U S A. 1994;91:10340-4.

28. Stagljar I, Korostensky C, Johnsson N, Te HS. A genetic system based on split-ubiquitin for the analysis of interactions between membrane proteins in vivo. Proc Natl Acad Sci U S A. 1998;95:5187-92.

29. Breeden L, Nasmyth K. Cell cycle control of the yeast Ho gene: cis- and trans-acting regulators. Cell. 1987;48:389-97.

30. Kaplan DR, Whitman M, Schaffhausen B, Pallas DC, White M, Cantley L, et al. Common elements in growth factor stimulation and oncogenic transformation: 85 kd phosphoprotein and phosphatidylinositol kinase activity. Cell. 1987;50:1021-9.

31. Eswar N, Eramian D, Webb B, Shen MY, Sali A. Protein structure modeling with modeller. Methods Mol Biol. 2008:426:145-59.

32. Humphrey W, Dalke A, Schulten K. Vmd: visual molecular dynamics. J Mol Graph. 1996;14:33-8

33. Holm L, Kaariainen S, Rosenstrom P, Schenkel A. Searching protein structure databases with Dalilite V. 3. Bioinformatics. 2008;24:2780-1.

34. Guex N, Peitsch MC. Swiss-Model and the Swiss-Pdb viewer: an environment for comparative protein modeling. Electrophoresis. 1997;18:2714-23.

35. Vriend G. What If: a molecular modeling and drug design program. J Mol Graph. 1990:8:52-6. 29

36. Morris AL, Macarthur MW, Hutchinson EG, Thornton JM. Stereochemical quality of protein structure coordinates. Proteins. 1992;12:345-64.

\section{Submit your next manuscript to BioMed Central and take full advantage of:}

- Convenient online submission

- Thorough peer review

- No space constraints or color figure charges

- Immediate publication on acceptance

- Inclusion in PubMed, CAS, Scopus and Google Scholar

- Research which is freely available for redistribution 